\title{
A MAXIMUM PRINCIPLE FOR COMPLETE HYPERSURFACES IN LOCALLY SYMMETRIC RIEMANNIAN MANIFOLD
}

\author{
Shicheng Zhang
}

\begin{abstract}
In this article, we apply the weak maximum principle in order to obtain a suitable characterization of the complete linear Weingarten hypersurfaces immersed in locally symmetric Riemannian manifold $N^{n+1}$. Under the assumption that the mean curvature attains its maximum and supposing an appropriated restriction on the norm of the traceless part of the second fundamental form, we prove that such a hypersurface must be either totally umbilical or hypersurface is an isoparametric hypersurface with two distinct principal curvatures one of which is simple.
\end{abstract}

\section{Introduction}

When the ambient manifolds possess very nice symmetry, for example the sphere, many results have been obtained in the study of the minimal hypersurface and hypersurface with constant mean curvature or constant scalar curvature in these ambient manifolds (One can see $[7,8,11,12,15,20,23]$ ). Recently, Q. M. Cheng and H. Nakagawa [6], and H. W. Xu [22] have proved the optimal rigidity theorem for hypersurface of constant mean curvature in a sphere independently.

In order to study hypersurface with constant scalar curvature, Cheng and Yau [9] introduced a new self-adjoint differential operator $\square$ acting on $C^{2}$ functions defined on Riemannian manifolds. As a by-product of this approach they were able to classify closed hypersurface $M$ with constant normalized scalar curvature $R$ satisfying $R \geq c$ and non-negative sectional curvatures immersed in complete and simply connected $(n+1)$-dimensional Riemannian manifolds of constant sectional curvature c, which will be denoted by $Q^{n+1}(c)$ and are also known as space forms.

By using the Cheng-Yau technique, X. Liu and H. Li [15] also obtained some rigidity theorems for hypersurface with constant scalar curvature. Therefore, it

Received May 9, 2013.

2010 Mathematics Subject Classification. 53B20, 53C40, 53C42.

Key words and phrases. locally symmetric, linear Weingarten hypersurfaces, totally umbilical. 
is important and natural to extend the Riemannian space forms to the locally symmetric Riemannian manifolds.

Let the ambient manifold $N^{n+1}$ be a locally symmetric Riemannian manifold with sectional curvature $K_{N}$ and $M$ is an $n$-dimensional complete hypersurface with constant mean curvature $H$ in $N^{n+1}$. When $\frac{1}{2}<\delta \leq K_{N} \leq 1$ ( $\delta$ is a constant) at all points $x \in M$ and the squared norm of the second fundamental form $S$ satisfies $S<n+\frac{n^{3}}{2(n-1) H^{2}}-\frac{n(n-2)}{2(n-1)} \sqrt{n^{2} H^{4}+4(n-1) H^{2}}$, S. Shu [21] and D. S. [10] has obtained the hypersurface $M$ is totally umbilical hypersurface, respectively. $\mathrm{H}$. W. $\mathrm{Xu}[24]$ has also obtained the same result when $M$ is an $n$-dimensional closed minimal hypersurface with constant mean curvature $H$ in $N^{n+1}$ and sectional curvature $K_{N}$ satisfying the condition $\delta \leq K_{N} \leq 1$ at all points $x \in M$ and the squared norm of the second fundamental form $S$ satisfies $S \leq(2 \delta-1) n$.

Next, Li et al. [14] extended the result of $[9,13]$ by considering linear Weingarten hypersurfaces immersed in the unit sphere $S^{n+1}(1)$, that is, hypersurfaces of $S^{n+1}(1)$ whose mean curvature $H$ and normalized scalar curvature $R$ satisfy $R=a H+b$ for some $a, b \in R$. In this setting, they showed that if $M$ is a compact linear Weingarten hypersurface with nonnegative sectional curvature immersed in $S^{n+1}(1)$, such that $R=a H+b$ with $(n-1) a^{2}+4 n(b-1) \geq 0$, then $M$ is either totally umbilical or isometric to a Clifford torus $S^{k}\left(\sqrt{1-r^{2}}\right) \times$ $S^{n-k}(r)$, where $1 \leq k \leq n-1$. By applying a weak Omori-Yau maximum principle, Pigola, Rigoli and Setti [17] studied the behavior of the scalar curvature $R$ of a complete hypersurface immersed with constant mean curvature into a real space form $Q_{c}^{n+1}$, deriving a sharp estimate for the infimum of $R$. Recently, Alías, García-Martínez and Rigoli [1] obtained another suitable weak maximum principle for complete hypersurfaces with constant scalar curvature in $Q_{c}^{n+1}$, and gave some applications of it in order to estimate the norm of the traceless part of its second fundamental form. In particular, they extended the main theorem of [3] for the context of $Q_{c}^{n+1}$.

Here, our purpose is to establish a new characterization theorem concerning the complete linear Weingarten hypersurfaces immersed in locally symmetric Riemannian manifold $N^{n+1}$. Now let us introduce a notion for linear Weingarten hypersurfaces in an $(n+1)$-dimensional locally symmetric Riemannian manifold $N^{n+1}$ as follows:

Definition 1.1. Let $M$ be a hypersurface in an $(n+1)$-dimensional locally symmetric Riemannian manifold $N^{n+1}$. We call $M$ a linear Weingarten hypersurface if $c R+d H+e=0$, where $c, d$ and $e$ are constants such that $c^{2}+d^{2} \neq 0$, $R$ and $H$, respectively, denote the scalar curvature and the mean curvature of $M$.

Remark 1.1. When the constant $d=0$ in Definition 1.1, a linear Weingarten hypersurface $M$ reduces to a hypersurface with constant scalar curvature. When the constant $c=0$ in Definition 1.1, a linear Weingarten hypersurface $M$ reduces to a hypersurface with constant mean curvature. In such a sense, the 
linear Weingarten hypersurfaces can be regarded as a natural generalization of hypersurfaces with constant scalar curvature or with constant mean curvature.

In this paper, let the ambient manifold $N^{n+1}$ be a locally symmetric Riemannian manifold with sectional curvature $K_{N}$ satisfying the condition

$$
\begin{aligned}
& K(u \wedge v) \geq c_{2}\left(c_{2} \text { is a constant }\right), \text { where } u, v \in T M \text { and } \\
& K(\omega \wedge v)=c_{1}\left(c_{1} \text { is a constant }\right), \text { where } \omega \in T^{\perp} M \text { and } v \in T M ;
\end{aligned}
$$

we shall say the manifold $N^{n+1}$ satisfies condition $(*)$. We study the linear Weingarten hypersurfaces in a locally symmetric Riemannian manifold.

Like in the computation of $([25,26])$, we compute the scalar curvature of a point in $N^{n+1}$,

$$
K=\sum_{A=1}^{n+1} K_{A A}=2 \sum_{i=1}^{n} K_{n+1 i n+1 i}+\sum_{i, j=1}^{n} K_{i j j i}=2 n c_{1}+\sum_{i, j=1}^{n} K_{i j j i} .
$$

It is known that $K$ is constant when $N^{n+1}$ is locally symmetric, so $\sum_{i, j=1}^{n} K_{i j j i}$ is constant.

From (2.3), we denote

$$
n(n-1) P=n^{2} H^{2}-S=n(n-1) r-\sum_{i, j=1}^{n} K_{i j j i},
$$

where $r$ is normalized scalar curvature of $M$. By investigating Cheng-Yau's operator $\square$ given in $[9]$ and using some new estimations, we want to study the linear Weingarten hypersurfaces in a locally symmetric Riemannian manifold as follows:

Theorem 1.1. Let $M$ be an $n(\geq 3)$-dimensional compact hypersurface in a locally symmetric Riemannian manifold $N^{n+1}$ satisfying condition $(*) . P=$ $a H+b, a, b \in \mathbb{R}$ and $(n-1) a^{2}+4 n b \geq 0, P>\frac{-2}{n} c$. The mean curvature $H$ attains its maximum. Let $\phi$ stand for the total umbilicity tensor of the immersion and if

Then

$$
\sup _{M}|\phi|^{2} \geq \alpha(n, P)=\frac{n(n-1)(P+c)^{2}}{(n-2)(n P+2 c)}>0 .
$$

(1) either $\sup _{M}|\phi|^{2}=0$ and $M$ is totally umbilical hypersurface; or

(2) $M$ is an isoparametric hypersurface with two distinct principal curvatures one of which is simple and $|\phi|^{2}=\alpha(n, P)$, where $c=2 c_{2}-c_{1} \geq 0$.

Remark 1.2. Since $n(n-1) P=n(n-1) r-\sum_{i, j=1}^{n} K_{i j j i}$, a hypersurface $M$ in Theorem 1.1 satisfying $r=a H+b$ is just a linear Weingarten hypersurface in Definition 1.1.

Remark 1.3. If $c_{1}=1, c_{2}=1$, i.e., the locally symmetric Riemannian manifold $N^{n+1}$ is the unit sphere $S^{n+1}(1)$, our Theorem 1.1 reduces to Theorem 1.1 in $[1]$. 
Remark 1.4. If $c_{1}=1, c_{2}=1$. When the constant $a$ in above identically vanishes, our Theorem 1.1 reduces to Theorem 2 in [2] and the main Theorem in $[5]$.

Remark 1.5. Equivalently, we can also state Theorem 1.1 in terms of the squared norm of the second fundamental form $S,(1)$ and (2) become:

If

$$
\sup _{M} S \geq \frac{(n-1)(n P+2 c)}{n-2}+\frac{(n-2) c^{2}}{n P+2 c},
$$

then

(1) either $\sup _{M} S=n P$ and $M$ is totally umbilical hypersurface; or

(2) $M$ is an isoparametric hypersurface with two distinct principal curvatures one of which is simple and $\sup _{M} S=\frac{(n-1)(n P+2 c)}{n-2}+\frac{(n-2) c^{2}}{n P+2 c}$.

Corollary 1.2. Let $M$ be an n-dimensional complete hypersurface immersed in a sphere $S^{n+1}(c), n \geq 3$, the normalized scalar curvature $r$ and the mean curvature $H$ of $M$ satisfy the following conditions: $P=r-c=a H+b, a, b \in \mathbb{R}$ and $(n-1) a^{2}+4 n b \geq 0, P>\frac{-2}{n} c$. The mean curvature $H$ attains its maximum. Let $\phi$ stand for the total umbilicity tensor of the immersion and if

$$
\sup _{M}|\phi|^{2} \geq \alpha(n, P)=\frac{n(n-1)(P+c)^{2}}{(n-2)(n P+2 c)}>0 .
$$

Then

(1) either $\sup _{M}|\phi|^{2}=0$ and $M$ is totally umbilical hypersurface; or

(2) $M$ is an isoparametric hypersurface with two distinct principal curvatures one of which is simple and $|\phi|^{2}=\alpha(n, P)$.

\section{Preliminaries}

If $M$ is a hypersurface with constant mean curvature in $N^{n+1}$. Let $\left\{e_{1}, e_{2}\right.$, $\left.\ldots, e_{n+1}\right\}$ be a local frame of orthonormal vector fields in $N^{n+1}$ such that, restricted to $M$ the vectors $\left\{e_{1}, e_{2}, \ldots, e_{n}\right\}$ are tangent to $M$, the vector $e_{n+1}$ is normal to $M$. Let $\left\{\omega^{1}, \omega^{2}, \ldots, \omega^{n+1}\right\}$ be its dual frame field. We use the following convention on the rang of indices:

$$
1 \leq A, B, C, \ldots \leq n+1,1 \leq i, j, k, \ldots \leq n .
$$

Let $K_{A B C D}$ and $R_{i j k l}$ be the components of the curvature tensors of $N^{n+1}$ and $M$ respectively. $h=h_{i j}$ be the second fundamental form of $M$, the square of the norm of $h$ is denoted by $S=\sum_{i, j=1}^{n}\left(h_{i j}\right)^{2}$.

It is well known that for an arbitrary hypersurface $M$ of $N^{n+1}$, we have

$$
\begin{gathered}
d \omega_{i j}=-\sum_{k} \omega_{i k} \wedge \omega_{k j}+\frac{1}{2} \sum_{k, l} R_{i j k l} \omega^{k} \wedge \omega^{l} . \\
R_{i j k l}=K_{i j k l}+h_{i k} h_{j l}-h_{i l} h_{j k} .
\end{gathered}
$$


Components $R_{i j}$ of Ricci tensor and the normalized scalar curvature $r$ of $M$ are given by

$$
\begin{array}{r}
R_{i j}=\sum_{k=1}^{n} K_{k i j k}+n H h_{i j}-\sum_{k=1}^{n} h_{i k} h_{k j}, \\
n(n-1) r=\sum_{i, j=1}^{n} K_{i j j i}+(n H)^{2}-S .
\end{array}
$$

We denote the first and second covariant derivatives of $h_{i j}$ by $h_{i j k}$ and $h_{i j k l}$ respectively, which are defined as in [7]. Following [7] and [16], we have

$$
\begin{gathered}
h_{i j k}-h_{i k j}=-K_{n+1 i j k} \\
\sum_{k} h_{i j k} \omega_{k}=d h_{i j}-\sum_{k} h_{i k} \omega_{k j}-\sum_{k} h_{k j} \omega_{k i}
\end{gathered}
$$

and the Ricci formula

$$
h_{i j k l}-h_{i j l k}=\sum_{s} h_{s j} R_{s i k l}+\sum_{s} h_{i s} R_{s j k l} .
$$

Let $K_{n+1 i j k, l}$ be the covariant derivative of $K_{n+1 i j k}$ as the section of $T^{\perp} M \otimes$ $T^{*} M \otimes T^{*} M \otimes T^{*} M$ and $K_{A B C D, E}$ be the covariant derivative of $K_{A B C D}$ as curvature tensor of $N^{n+1}$. Restricted to $M$ we have

$$
\sum_{l} K_{n+1 i j k l} \omega_{l}=d K_{n+1 i j k}+\sum_{s} K_{n+1 s j k} \omega_{i s}+\sum_{s} K_{n+1 i j s} \omega_{k s}
$$

and

(2.8) $K_{n+1 i j k, l}=K_{n+1 i j k l}-K_{n+1 i n+1 k} h_{j l}-K_{n+1 i j n+1} h_{k l}+\sum_{m} K_{m i j k} h_{m l}$.

The mean curvature of $M$ is given by $H=\frac{1}{n} \sum_{i=1}^{n} h_{i i} e_{n+1}$, the Laplacian $\Delta h_{i j}$ of the second fundamental form $h$ of $M$ is defined by $\Delta h_{i j}=\sum_{k=1}^{n} h_{i j k k}$ $\Delta h_{i j}=\sum_{k} h_{k k i j}+n H K_{n+1 i n+1 j}-\sum_{k} K_{n+1 k n+1 k} h_{i j}+n H \sum_{k} h_{i k} h_{k j}-S h_{i j}$

$$
+\sum_{k}\left(K_{l k i k} h_{l j}+K_{l k j k} h_{l i}+2 K_{l i j k} h_{l k}\right)-\sum_{k}\left(K_{n+1 i j k, k}+K_{n+1 k i k, j}\right) .
$$

Since $N^{n+1}$ is complete and locally symmetric, thus

$$
K_{A B C D, E}=0
$$

for all $A, B, C, D, E$. This together with (2.2), (2.7) and (2.8) implies

$$
\begin{aligned}
\frac{1}{2} \Delta S & =\sum_{i, j, k} h_{i j k}^{2}+\sum_{i, j, k} h_{i j k k} h_{i j} \\
& =n H \operatorname{tr} H_{n+1}^{3}-S^{2}+\sum_{i, j, k} h_{i j k}^{2}+\sum_{i, j, k, l} 2\left(h_{i j} h_{k l} K_{l i j k}+h_{l i} h_{i j} K_{l k j k}\right)
\end{aligned}
$$




$$
+\sum_{i, j, k} h_{i j k k} h_{i j}+\sum_{i, j} n H h_{i j} K_{n+1 i j n+1}-S \sum_{k} K_{n+1 k n+1 k} .
$$

Following Cheng-Yau [9], we introduce a modified operator $\square$ acting on any $C^{2}$-function $f$ by

$$
L(f)=\left[\square-\frac{n-1}{2} a \Delta\right] f=\sum_{i, j}\left(n H \delta_{i j}-h_{i j}\right) f_{i j}-\frac{n-1}{2} a \Delta f .
$$

Choose a local frame of orthonormal vector fields $\left\{e_{i}\right\}$ in order to at arbitrary point $x$ of $M h_{i j}=\lambda_{i} \delta_{i j}$, then at the point $x$, and by use of (1.1) and (2.10), we have

$$
\begin{aligned}
L(n H)= & n H \Delta(n H)-\sum_{l} \lambda_{i}(n H)_{i i}-\frac{n-1}{2} a \Delta(n H) \\
= & \frac{1}{2} \Delta\left(n(n-1) r-\sum_{i, j=1}^{n} K_{i j j i}\right)+\frac{1}{2} \Delta S \\
& -n^{2}|\nabla H|^{2}-\sum_{i} \lambda_{i}(n H)_{i i}-\frac{1}{2} \Delta(n(n-1) r) \\
= & \frac{1}{2} \Delta S-n^{2}|\nabla H|^{2}-\sum_{i} \lambda_{i}(n H)_{i i} .
\end{aligned}
$$

Putting (2.9) into (2.11), we obtain

$$
\begin{aligned}
L(n H) & =n H \Delta(n H)-\sum_{l} \lambda_{i}(n H)_{i i} \\
2.13) & =\frac{1}{2} \Delta\left(n(n-1) r-\sum_{i, j=1}^{n} K_{i j j i}\right)+\sum_{i, j, k} h_{i j k}^{2}-n^{2}|\nabla H|^{2}+X+Y+Z,
\end{aligned}
$$

where

$$
\begin{aligned}
X & =n H \operatorname{tr} H_{n+1}^{3}-S^{2}, \\
Y & =\sum_{i, j, k, l} 2\left(h_{i j} h_{k l} K_{l i j k}+h_{l i} h_{i j} K_{l k j k}\right), \\
Z & =\sum_{i, j} n H h_{i j} K_{n+1 i j n+1}-S \sum_{k} K_{n+1 k n+1 k} .
\end{aligned}
$$

\section{Proof of Theorem 1.1}

The following lemmas are useful in the proof of Theorem 1.1.

Lemma 3.1. Let $M$ be an $n$-dimensional hypersurface in a locally symmetric Riemannian manifold $N^{n+1}$ satisfying condition $(*)$. If $P=a H+b, a, b \in \mathbb{R}$ and $(n-1) a^{2}+4 n b \geq 0$. Then we have

$$
|\nabla h|^{2}=\sum_{\alpha, i, j, k}\left(h_{i j k}^{\alpha}\right)^{2} \geq n^{2}|\nabla H|^{2} .
$$


Proof. From Gauss equation, we have

$$
S=n^{2} H^{2}-n(n-1) P=n^{2} H^{2}-n(n-1)(a H+b) .
$$

Taking the covariant derivative of the above equation, we have

$$
2 \sum_{\alpha, i, j} h_{i j}^{\alpha} h_{i j k}^{\alpha}=2 n^{2} H H_{k}-n(n-1) a H_{k} .
$$

Therefore,

$$
4|h|^{2}|\nabla h|^{2} \geq 4 \sum_{k}\left(h_{i j}^{\alpha} h_{i j k}^{\alpha}\right)^{2}=\left[2 n^{2} H-n(n-1) a\right]^{2}|\nabla H|^{2} .
$$

On the other hand,

$$
\begin{aligned}
& {\left[2 n^{2} H-n(n-1) a\right]^{2}-4 n^{2} S } \\
= & 4 n^{4} H^{2}+n^{2}(n-1)^{2} a^{2}-4 n^{3}(n-1) H a \\
& -4 n^{3}\left[n H^{2}-(n-1)(a H+b)\right] \\
= & n^{2}(n-1)\left[(n-1) a^{2}+4 n b\right] \\
\geq & 0,
\end{aligned}
$$

it follows that

$$
|\nabla h|^{2} \geq n^{2}|\nabla H|^{2}
$$

Lemma $3.2([16])$. Let $\mu_{1}, \ldots, \mu_{n}$ be real numbers such that $\sum_{i} \mu_{i}=0$ and $\sum_{i} \mu_{i}^{2}=B$, where $B=$ const. $\geq 0$, then

$$
\left|\sum_{i} \mu_{i}^{3}\right| \leq \frac{n-2}{\sqrt{n(n-1)}} B^{\frac{3}{2}}
$$

and equality holds if and only if

$$
\mu_{1}=\cdots=\mu_{n-1}=-\sqrt{\frac{1}{n(n-1)} B}, \mu_{n}=\sqrt{\frac{n-1}{n} B} .
$$

Choose a local frame of orthonormal vector fields $\left\{e_{i}\right\}$ in order to at arbitrary point $x$ of $M h_{i j}=\lambda_{i} \delta_{i j}$, then at the point $x$ we have

$$
S=\sum_{i} \lambda_{i}^{2}
$$

Putting $\mu_{j}=H-\lambda_{j}$, we obtain

$$
\sum_{j} \mu_{j}=0, \quad|\phi|^{2}=\sum_{j} \mu_{j}^{2}=S-n H^{2},
$$

and

$$
\sum_{i} \lambda_{i}^{3}=n H^{3}+3 H \sum_{i} \mu_{i}^{2}-\sum_{i} \mu_{i}^{3} .
$$


So

$$
\begin{aligned}
n H \operatorname{tr} H_{n+1}^{3} & =n H\left(n H^{3}+3 H \sum_{i} \mu_{i}^{2}-\sum_{i} \mu_{i}^{3}\right) \\
& \geq 3 n H^{2}|\phi|^{2}+n^{2} H^{4}-n|H| \frac{n-2}{\sqrt{n(n-1)}}|\phi|^{3}
\end{aligned}
$$

and we obtain

Lemma 3.3. $X \geq|\phi|^{2}\left[n H^{2}-|\phi|^{2}-\frac{n(n-2)}{\sqrt{n(n-1)}}|H||\phi|\right]$.

Lemma $3.4([24]) . \quad Y \geq 2 n c_{2}|\phi|^{2}$.

Lemma 3.5. $Z=c_{1} n\left(n H^{2}-S\right)=-c_{1} n|\phi|^{2}$.

Proof.

$$
\begin{aligned}
Z & =\sum_{i, j} n H h_{i j} K_{n+1 i j n+1}-S \sum_{k} K_{n+1 k n+1 k} \\
& =n H \sum_{i} \lambda_{i} K_{n+1 i n+1 i}-S \sum_{i} K_{n+1 i n+1 i} \\
& =\sum_{i}\left(S-n H \lambda_{i}\right) K_{n+1 i n+1 i}=c_{1} n\left(n H^{2}-S\right) .
\end{aligned}
$$

This proves Lemma 3.5.

From the assumption of Theorem that $\sum_{i, j=1}^{n} K_{i j j i}=$ const. and Lemmas $3.1,3.3,3.4$ and 3.5 , we obtain

$$
L(n H) \geq|\phi|^{2}\left[n c-|\phi|^{2}-n|H| \frac{n-2}{\sqrt{n(n-1)}}|\phi|+n H^{2}\right],
$$

where $c=2 c_{2}-c_{1}$.

Recall that $|\phi|^{2}=S-n H^{2}$, so that (3.3) becomes

$$
\frac{1}{2(n-1)} L\left(|\phi|^{2}\right) \geq H|\phi|^{2}\left[n c-|\phi|^{2}-n|H| \frac{n-2}{\sqrt{n(n-1)}}|\phi|+n H^{2}\right] .
$$

On the other hand, we have

$$
H^{2}=\frac{1}{n(n-1)}|\phi|^{2}+P,
$$

and therefore, taking into account that $H \geq 0$, we may write

$$
H=\frac{1}{\sqrt{n(n-1)}} \sqrt{|\phi|^{2}+n(n-1) P}
$$

Finally, we obtain

$$
\frac{1}{2} L\left(|\phi|^{2}\right) \geq \frac{1}{\sqrt{n(n-1)}}|\phi|^{2} Q_{P}(|\phi|) \sqrt{|\phi|^{2}+n(n-1) P},
$$

where

(3.6) $Q_{P}(x)=-(n-2) x^{2}-(n-2) x \sqrt{x^{2}+n(n-1) P}+n(n-1)(P+c)$. 
Lemma 3.6. Let $M$ be an $n$-dimensional compact hypersurface in a locally symmetric Riemannian manifold $N^{n+1}$ satisfying condition $(*)$ such that $P=$ $a H+b$ and $(n-1) a^{2}+4 n b \geq 0$, where $a$ and $b$ are constants. Let $\mu_{-}$and $\mu_{+}$ be the minimum and the maximum of the eigenvalues of $L$, respectively. Then

$$
\mu_{-}>0, \mu_{+}<2 n H-(n-1) a .
$$

Proof. From (1.1), we have

$$
0 \leq S=n^{2} H^{2}-n(n-1) P=n^{2} H^{2}-n(n-1)(a H+b),
$$

thus, $H$ satisfies

$$
\begin{aligned}
& H \geq \frac{(n-1) a+\sqrt{(n-1)^{2} a^{2}+4 n(n-1) b}}{2 n} \text { or } \\
& H \leq \frac{(n-1) a-\sqrt{(n-1)^{2} a^{2}+4 n(n-1) b}}{2 n},
\end{aligned}
$$

we get

$$
\begin{aligned}
& n H-\frac{(n-1) a}{2} \geq \frac{\sqrt{(n-1)^{2} a^{2}+4 n(n-1) b}}{2} \text { or } \\
& n H-\frac{(n-1) a}{2} \leq \frac{\sqrt{(n-1)^{2} a^{2}+4 n(n-1) b}}{2} .
\end{aligned}
$$

This means that $n H-\frac{(n-1) a}{2}$ has the same sign on $M$. Without loss of generality, we assume $n H-\frac{(n-1) a}{2}>0$.

Since

$$
\begin{aligned}
{\left[n H-\frac{(n-1) a}{2}\right]^{2} } & =\frac{1}{4}\left[4 n^{2} H^{2}-4 n(n-1) a H+(n-1)^{2} a^{2}\right] \\
& >n^{2} H^{2}-n(n-1) a H-n(n-1) b \\
& =S \geq\left(\lambda_{i}\right)^{2}, \forall i,
\end{aligned}
$$

moreover, $\mu_{i}=n H-\frac{(n-1) a}{2}-\lambda_{i}$ are precisely the eigenvalues of the operator $L$, we obtain $\mu_{-} \geq 0$ and $\mu_{+} \leq 2 n H-(n-1) a$ in particular.

Remark 3.1. From Lemma 3.6, which implies $L$ is elliptic.

In what follows we shall also need a version of a weak maximum principle for the operator $L$ that we state in a form useful to our present purposes.

Theorem $3.7([1,18])$. Let $(\Sigma,\langle\rangle$,$) be a complete Riemannian manifold, let$ $o$ be a reference point in $\Sigma$, and let $r(p)$ be the distance function from o. Let $h$ be a symmetric $(0,2)$ tensor field on $\Sigma$ and set $\sharp: T^{*} \Sigma \rightarrow T \Sigma$ to denote the musical isomorphism, so that $h(X,)^{\#}$ is the vector field on $\Sigma$ defined by

$$
\left\langle h(X, \cdot)^{\sharp}, Y\right\rangle=h(X, Y)
$$

for every $Y \in T_{p} \Sigma$ and $p \in \Sigma$. Assume that, for some positive continuous function $h_{+}$defined on $[0,+\infty)$, the tensor $h$ satisfies the following bound

$$
0 \leq h(X, Y) \leq h_{+}(r)
$$


for every $X \in T_{p} \Sigma,|X|=1$, and every $p \in \partial B_{r}$, where $B_{r}$ denotes the geodesic ball of radius $r$ centered at $o$. Set

$$
h_{+}^{*}(r)=\sup _{s \leq r} h_{+}(s) .
$$

Given $f \in C^{0}(R)$, assume that $u \in C^{2}(\Sigma)$ satisfies $u^{*}=\sup _{\Sigma} u<+\infty$ and

$$
\mathcal{L}(u)=\operatorname{div}\left(h(\nabla u, \cdot)^{\sharp}\right) \geq f(u)
$$

on the set $\Omega_{\gamma}=\{p \in \Sigma: u(p)>\gamma\}$ for some $\gamma<u^{*}$. If

$$
\lim _{r \rightarrow+\infty} \frac{h_{+}^{*}(r)}{r^{2}}=0
$$

and

$$
\liminf _{r \rightarrow+\infty} \frac{h_{+}^{*}(r) \log \operatorname{vol} B_{r}}{r^{2}}<+\infty
$$

then $f\left(u^{*}\right) \leq 0$.

As we shall see in the next section, the idea of proof of our main results is to apply Theorem 3.7 to the operator $L$, by considering the operator $h$ given by

$$
h(X, Y)\langle P X, Y\rangle
$$

for every $u \in C^{2}$. Thus, $\left.\mathcal{L}(u)=\operatorname{div}\left(h(\nabla u)-\frac{n-1}{2} a \nabla u, \cdot\right)^{\sharp}\right)=L(u)$. Toward this aim, from Lemma 3.6 and under the hypothesis of Theorem 1.1, we have

$$
h_{+}(r)=2 n \sup _{\partial B_{r}} H-(n-1) a,
$$

so that

$$
h_{+}^{*}(r)=2 n \sup _{B_{r}} H-(n-1) a .
$$

On the other hand, conditions (3.7) and (3.8) are also satisfied if $\sup _{\Sigma}|\phi|^{2}<$ $+\infty$. Since $P=a H+b$, $\sup _{\Sigma}|\phi|^{2}<+\infty$ implies that $\sup _{\Sigma} H<+\infty$. Thus

$$
h_{+}^{*}(r)=2 n \sup _{B_{r}} H-(n-1) a \leq 2 n \sup _{\Sigma} H-(n-1) a
$$

and therefore (3.7) is automatically satisfied. As for (3.8), by (3.9) it becomes

$$
\liminf _{r \rightarrow+\infty} \frac{\log \operatorname{vol} B_{r}}{r^{2}}<+\infty .
$$

From $\sup _{\Sigma}|\phi|^{2}<+\infty$, we also have that $\sup _{\Sigma} S=\sup _{\Sigma}|A|^{2}<+\infty$. Thus, we can estimate

$$
H\langle A X, X\rangle \geq-\sup _{\Sigma} H\langle A X, X\rangle \geq-\sup _{\Sigma} H \sup _{\Sigma}|A||X|^{2},
$$

and

$$
|A X|^{2} \leq \sup _{\Sigma}|A|^{2}|X|^{2}
$$


for $X \in T \Sigma$, we get

$$
\begin{aligned}
\operatorname{Ric}(X, X) & =(n-1)|X|^{2}+n H\langle A X, X\rangle-|A X|^{2} \\
& \geq\left[(n-1) c_{2}-n \sup _{\Sigma} H \sup _{\Sigma}|A|-\Sigma|A|^{2}\right]|X|^{2} .
\end{aligned}
$$

Therefore, we obtain that the Ricci curvature of $\Sigma$ is bounded from below. Since $\Sigma$ is complete, by Bishops volume comparison theorem it follows that (3.10) is satisfied. As a consequence, we obtain the following weak maximum principle for $L$.

Corollary 3.8. Let $M \rightarrow N^{n+1}$ be a complete oriented isometrically immersed hypersurface with $P=a H+b,(n-1) a^{2}+4 n b \geq 0$ and $H$ attains its maximum on $M$. Assume that $\sup _{M}|\phi|^{2}<+\infty$. If $u \in \bar{C}^{2}(M)$ satisfies $u^{*}=\sup _{M} u<$ $+\infty$ and for a given $f \in C^{0}(R), L(u) \geq f(u)$ on the set $\Omega_{\gamma}=\{p \in M: u(p)>$ $\gamma$ for some $\gamma<u^{*}$, then $f\left(u^{*}\right) \leq 0$.

Next, we will prove the main theorem.

If $\sup _{M}|\phi|^{2}=+\infty$, then (ii) of Theorem 1.1 is trivially satisfied and there is nothing to prove. If $\sup _{M}|\phi|^{2}=0$, then (i) holds. Then let us assume that $0<\sup _{M}|\phi|^{2}<+\infty$. This can be proved after a similar argument as in [1], we omit the details here.

Since

$$
H^{2}=\frac{1}{n(n-1)}|\phi|^{2}+P .
$$

Thus, from (3.5) and (3.11). Let $u=|\phi|^{2}$, we obtain

$$
L(u) \geq \frac{2}{\sqrt{n(n-1)}} u Q_{P}(\sqrt{u}) \sqrt{u+n(n-1) P}=f(u),
$$

where

$$
Q_{P}(x)=-(n-2) x^{2}-(n-2) x \sqrt{x^{2}+n(n-1) P}+n(n-1)(P+c) .
$$

Therefore, by applying Corollary 3.1, the inequality (3.12), we have

$$
f\left(u^{*}\right) \leq 0,
$$

from the inequality, we get

$$
Q_{P}\left(\sqrt{u^{*}}\right) \leq 0 \text {. }
$$

Since we are supposing that $P>\frac{-2}{n} c, P_{R}(0)=n(n-1)(P+c)>0$ and the function $Q_{P}(x)$ is strictly decreasing for $x \geq 0$, with $Q_{P}\left(x_{0}\right)=0$ at

$$
x_{0}=(P+c) \sqrt{\frac{n(n-1)}{(n-2)(n P+2 c)}}>0,
$$

therefore, we obtain

$$
\sup _{M}|\phi|^{2} \geq \frac{n(n-1)(P+c)^{2}}{(n-2)(n P+2 c)} .
$$


Moreover, equality $\sup _{M}|\phi|^{2}=\frac{n(n-1)(P+c)^{2}}{(n-2)(n P+2 c)}$ holds if and only if $\sqrt{u^{*}}=x_{0}$, and $Q_{P}(\sqrt{u}) \geq 0$ on $M$, jointly with (3.5) implies that

$$
L(u) \geq 0 \text { on } M .
$$

Consequently, since $L$ is elliptic and as we are supposing that $H$ attains its maximum on $M$, from (3.14) we conclude that $H$ is constant on $M$. Thus, we get

$$
|\nabla A|^{2}=n^{2}|\nabla H|^{2}=0
$$

and it follows that $\lambda_{i}$ is constant for every $i=1, \ldots, n$. Hence, by the classical results on isoparametric hypersurfaces of real space forms [4, 19] and since we are supposing $P>0$, we conclude that either $|\phi|=0$ and $M$ is totally umbilical, or

$$
|\phi|^{2}=\frac{n(n-1)(P+c)^{2}}{(n-2)(n P+2 c)} .
$$

If $M$ is not totally umbilical, we can see that the equalities hold in Lemma 3.1 and Lemma 3.2, and it follows that $\lambda_{i}=$ const. for all $i$ and $(n-1)$ of the $\lambda_{i}^{\prime} s$ are equal. After re-number if necessary, we can assume that

$$
\lambda_{1}=\lambda_{2}=\cdots=\lambda_{n-1}, \lambda_{1} \neq \lambda_{n} .
$$

Therefor, $M$ is a isoparametric hypersurface with two distinct principal curvatures one of which is simple.

This completes the proof of Theorem 1.1.

Acknowledgements. This subject is supported by the National Natural Science Foundation of China (No.61271002) and the Project Funded by the Priority Academic Program Development (PAPD) of Jiangsu Higher Education Institutions.

\section{References}

[1] L. J. Alías, S. C. García-Martínez, and M. Rigoli, A maximum principle for hypersurfaces with constant scalar curvature and applications, Ann. Global Anal. Geom. 41 (2012), no. 3, 307-320.

[2] A. Brasil Jr., A. G. Colares, and O. Palmas, Complete hypersurfaces with constant scalar curvature in spheres, Monatsh. Math. 161 (2010), no. 4, 369-380.

[3] - Complete hypersurfaces with constant scalar curvature in spheres, Monatsh. Math. 161 (2010), no. 4, 369-380.

[4] É. Cartan, Familles de surfaces isoparamétriques dans les espaces à courbure constante, Ann. Mat. Pura Appl. 17 (1938), no. 1, 177-191.

[5] Q. M. Cheng, Hypersurfaces in a unit sphere $S^{n+1}$ with constant scalar curvature, J. London Math. Soc. (2) 64 (2001), no. 3, 755-768.

[6] Q. M. Cheng and H. Nakagawa, Totally umbilic hypersurfaces, Hiroshima Math. J. 20 (1990), no. 1, 1-10.

[7] Q. M. Cheng and Susumu, Characterization of the clifford torus, Proc. Amer. Math. Soc. 127 (1999), no. 3, 819-831. 
[8] S. S. Cheng, M. do Carmo, and S. Kobayashi, Minimal submanifolds of a sphere with second fundamental form of constant length, in Functional Analysis and Related Fields (Proc. Conf. for M. Stone, Univ. Chicago, Chicago, Ill., 1968), 59-75, Springer, New York, 1970.

[9] S. Y. Cheng and S. T. Yau, Hypersurfaces with constant scalar curvature, Math. Ann. 225 (1977), no. 3, 195-204.

[10] S. H. Ding and J. F. Zhang, Hypersurfaces in a locally symmetric manifold with constant mean curvature, Pure Appl. Math. 22 (2006), no. 1, 94-99.

[11] Z. H. Hou, Hypersurfaces in a sphere with constant mean curvature, Proc. Amer. Math. Soc. 125 (1997), no. 4, 1193-1196.

[12] H. B. Lawson, Jr., Local rigidity theorems for minimal hypersurfaces, Ann. of Math. 89 (1969), no. 2, 187-197.

[13] H. Li, Global rigidity theorems of hypersurfaces, Ark. Mat. 35 (1997), no. 2, 327-351.

[14] H. Li, Y. Suh, and G. Wei, Linear Weingarten hypersurfaces in a unit sphere, Bull. Korean Math. Soc. 46 (2009), no. 2, 321-329.

[15] X. X. Liu and H. Li, Complete hypersurfaces with constant scalar curvature in a sphere, Comment. Math. Univ. Carolin. 46 (2005), no. 3, 567-575.

[16] M. Okumura, Hypersurfaces and a pinching problem on the second fundamental tensor, Amer. J. Math. 96 (1974), no. 4, 207-213.

[17] S. Pigola, M. Rigoli, and A. G. Setti, Maximum principles on Riemannian manifolds and applications, Mem. Amer. Math. Soc. 174 (2005), no. 822, x+99 pp.

[18] _ A Liouville-type result for quasi-linear elliptic equations on complete Riemannian manifolds, J. Funct. Anal. 219 (2005), no. 2, 400-432.

[19] B. Segre, Famiglie di ipersuperficie isoparametriche negli spazi euclidei ad un qualunque numero di dimensioni, Atti Accad. Naz. Lincei, Rend., VI. Ser. 27 (1938), 203-207.

[20] J. Simons, Minimal varieties in Riemannian manifolds, Ann. of Math. 88 (1968), no. $2,62-105$.

[21] S. C. Shu and S. Y. Liu, Complete hypersurfaces with constant mean curvature in locally symmetric manifold, Adv. Math. (China) 33 (2004), no. 5, 563-569.

[22] H. W. Xu, Pinching theorems, global pinching theorems and eigenvalues for Riemannian submanifolds, Ph. D. dissertation, Fudan University, 1990.

[23] _ On closed minimal submanifolds in pinched Riemannian manifolds, Trans. Amer. Math. Soc. 347 (1995), no. 5, 1743-1751.

[24] H. W. Xu and X. Ren, Closed hypersurfaces with constant mean curvature in a symmetric manifold, Osaka J. Math. 45 (2008), no. 3, 747-756.

[25] S. Zhang and B. Wu, Rigidity theorems for complete spacelike hypersurfaces with constant scalar curvature in locally symmetric Lorentz spaces, J. Geom. Phys. 60 (2010), no. $2,333-340$.

[26] _ Complete hypersurfaces with constant mean curvature in a locally symmetric Riemannian manifold, Acta Math. Sci. Ser. A Chin. Ed. 30 (2010), no. 4, 1000-1005.

School of Mathematics and Statistics

JiANGSU NORMAL UNIVERSITY

Xuzhou, Jiangsu 221116, P. R. China

E-mail address: zhangshicheng@jsnu.edu.cn 